\title{
Electron impact excitation cross sections for carbon monoxide
}

\author{
Lee Mu-Taot and Vincent McKoy
}

A A Noyes Laboratory of Chemical Physics $\$$, California Institute of Technology, Pasadena, California 91125 , USA

Received 19 April 1982

\begin{abstract}
We present distorted-wave cross sections for excitation of the $\mathrm{A}{ }^{1} \Pi, \mathrm{a}^{3} \Pi, \mathrm{a}^{\prime}{ }^{3} \Sigma^{+}$, $\mathrm{D}^{1} \Delta$ and $\mathrm{d}^{3} \Delta$ states of $\mathrm{CO}$ by electrons in the 20 to $50 \mathrm{eV}$ energy range. In these studies both the initial and final distorted waves are obtained in the static-exchange field of the ground electronic state. Differential and integral cross sections are presented and compared with available experimental data and with other calculations. The calculated differential cross sections for the $\mathrm{A}^{1} \Pi$ and $\mathrm{a}^{3} \Pi$ states agree poorly in magnitude, but better in shape, with available experimental data. In general the distorted-wave cross sections differ substantially from those of plane-wave-type theories such as the BornOchkur-Rudge approximation.
\end{abstract}

\section{Introduction}

The electronic excitation of molecules by electrons in the low and intermediate energy range is a very important process in many fields of physics and chemistry. However, to date, there has been limited progress in the experimental determination of these cross sections (Cartwright et al 1977a, b, Chutjian et al 1977, 1983, Trajmar et al 1968, 1971, Finn and Doering 1976, Linder and Schmidt 1971) as well as in the development of new theoretical methods (Chung and Lin 1978, Holley et al 1981, Rescigno et al 1975, 1976, Fliflet and McKoy 1980, Hazi 1981) for studying these cross sections. Until quite recently, Born-type approximations (Arrighini et al 1980, Chung and Lin 1972, 1974, Chung et al 1975, Cartwright 1970, 1972, Khare 1966, $1967)$ were the only practical method for calculating these inelastic cross sections. On the experimental side, $\mathrm{N}_{2}$ is the only molecule for which the electronic excitation cross sections have been extensively studied at low and intermediate energies (Cartwright et al 1977a, b, Chutjian et al 1977, 1983, Finn and Doering 1976). In view of the significant experimental effort and difficulties involved in the measurement of these cross sections for molecules, it is clearly of interest to develop theoretical methods with some degree of reliability which can be applied routinely at least for linear molecules.

Recently, we have used the distorted-wave method (DW) to study the electronic excitation cross sections in $\mathrm{H}_{2}$ (Lee et al 1982) and $\mathrm{N}_{2}$ (Lee and McKoy 1982a, b).

$\ddagger$ Permanent address: Departamento de Quimica, Universidade Federal de São Carlos, São Carlos, 13560 , SP Brasil.

¥Contribution No 6608 . 
The results were encouraging in light of their comparison with available experimental data. The DW integral cross sections (Lee et al 1982) as well as those of Fliflet and McKoy (1980) agreed quite well with the available experimental data for excitation of the $b^{3} \Sigma_{u}^{+}$(Corrigan 1965), B ${ }^{1} \Sigma_{u}^{+}$(Srivastava and Jensen 1977) and $C^{1} \Pi_{u}$ (Yung et al 1982) states. The DW integral cross sections (Fliflet and McKoy 1980) were similar to the two-state close-coupling (CC) results of Chung et al (1975) for the B ${ }^{1} \Sigma_{u}^{+}$ state but differed substantially from their CC cross sections for the $b^{3} \Sigma_{u}^{+}$. In the case of $\mathrm{N}_{2}$, the method successfully reproduced the shape and magnitude of the experimental differential inelastic cross sections for excitation of several states. Such studies also serve to reveal the limitations of the DW method and to identify states where the assumptions of the distorted-wave method may fail.

In this paper, we report the results of similar distorted-wave studies for electronic excitation of $\mathrm{CO}$. These studies can provide a further test of this method. Moreover, a comparison of the cross sections for $\mathrm{CO}$ and $\mathrm{N}_{2}$ can be useful. Cross sections for low-energy electron-CO scattering are also required for modelling plasmas containing $\mathrm{CO}$, as well as in gas-discharge lasers and in coal-fired MHD generators (Land 1978). These results are also useful in interpreting observations of dayglow emissions from the upper atmosphere of Mars and Venus.

Electronic excitation cross sections for several states of $\mathrm{CO}$ have been calculated by Chung and Lin (1974) using the Born-Ochkur-Rudge (BOR) approximation. Experimentally, some differential cross sections (DCS) in the low-energy range have been measured by Rice (1968) and by Cartwright and Trajmar (1981). Integral cross sections for excitation of the $\mathrm{A}^{1} \Pi$ (Ajello 1971, Aarts and de Heer 1970, Mumma et al 1971) and $\mathrm{a}^{3} \Pi$ (Ajello 1971) states have been measured by the emission method. Integral cross sections for excitation of several electronic excited states have also been estimated by the semi-empirical method (Sawada et al 1972).

In this paper, we present the DW cross sections for excitation of the $A^{1} \Pi, a^{3} \Pi$, $\mathrm{a}^{\prime}{ }^{3} \Sigma^{+}, \mathrm{D}^{1} \Delta$ and $\mathrm{d}^{3} \Delta$ states in the $20-50 \mathrm{eV}$ energy range. In $\S 2$, we briefly outline the relevant theory. Details of the calculations and comparisons with the available experimental and theoretical data are presented in $\S 3$.

\section{Theory}

Using the Born-Oppenheimer and Franck-Condon approximations, and treating the target rotational levels as essentially degenerate, the differential cross sections for electronic excitation by electron impact can be written as:

$\frac{\mathrm{d} \sigma}{\mathrm{d} \Omega}\left(n \leftarrow 0 ; E, \hat{r}^{\prime}\right)=S M_{n} \sum_{v^{\prime}} \frac{k_{\nu^{\prime}}}{k_{0}} q_{\nu^{\prime} 0} \frac{1}{8 \pi^{2}} \int \mathrm{d} \hat{\boldsymbol{R}}^{\prime}\left|f_{k_{0}}\left(n \leftarrow 0 ; \boldsymbol{R}^{\prime}, \hat{r}^{\prime}\right)\right|^{2}$

where $E$ is the impact energy, $k_{0}$ and $k_{\nu^{\prime}}$ the momenta of the incoming and outgoing electrons, respectively, $q_{\nu^{\prime} 0}$ the Franck-Condon factor for the $\nu=0$ level of the initial electronic state and $\nu^{\prime}$ the vibrational level of the excited state. The factor $S$ results from summing over final levels and averaging over initial spin sublevels. For singlet excitations $S$ equals $\frac{1}{2}$ and for singlet to triplet excitations $S$ equals $\frac{3}{2}$. For linear molecules $M_{n}$ is the orbital angular momentum projection degeneracy factor of the final state ( 1 for a $\Sigma$ state and 2 for a $\Pi$ state). The laboratory-frame scattering 
amplitude $f_{k_{0}}$ can be related to the fixed-nuclei dynamical coefficients as follows:

$f_{k_{0}}\left(n \leftarrow 0 ; \boldsymbol{R}^{\prime}, \hat{r}^{\prime}\right)=\sum_{l l^{\prime} m m^{\prime}} a_{l l^{\prime} m m^{\prime}}\left(n \leftarrow 0 ; k_{0}, R\right) D_{m^{\prime} m}^{\left(l^{\prime}\right)^{*}}(\hat{R}) D_{o m^{\prime}}^{\left(l^{\prime}\right)}(\hat{R}) Y_{l m^{\prime \prime}}\left(\hat{r}^{\prime}\right)$

where $D_{m v m}^{\left(l^{\prime}\right)}\left(\hat{R}^{\prime}\right)$ is the rotational harmonic and the fixed-nuclei dynamical coefficients, $a_{l l^{\prime} m m^{\prime}}$, can be approximated by the fixed-nuclei partial-wave components of the electronic portion of the DW transition matrix elements as:

$a_{l l^{\prime} m m^{\prime}}\left(n \leftarrow 0, k_{0}, R\right)=-\frac{1}{2} \pi\left[4 \pi\left(2 l^{\prime}+1\right)\right]^{1 / 2} \mathrm{i}^{l-l^{\prime}}\left\langle k_{n} l m, n\left|T_{\mathrm{el}}\right| k_{0} l^{\prime} m^{\prime}, 0\right\rangle$

where $\left\langle k_{n} l m, n\left|T_{\mathrm{el}}\right| k_{0} l^{\prime} m^{\prime}, 0\right\rangle$ is the DW transition matrix element. In this DW approach, $\left|k_{n} l m\right\rangle$ and $\left|k_{0} l^{\prime} m^{\prime}\right\rangle$ represent partial-wave components of the final and initial HartreeFock continuum spin orbitals of the ground-state potential respectively and $n$ and 0 designate the excited and initial electronic states of the target. Further details are discussed by Fliflet and McKoy (1980).

\section{Calculations and results}

In this work, the elastic static-exchange continuum wavefunctions are obtained by the iterative Schwinger variational method (Lucchese and McKoy 1979, Watson et al 1980). The static-exchange potential is generated using the ground-state SCF wavefunction of $\mathrm{CO}$, with the electronic configuration of $1 \sigma^{2} 2 \sigma^{2} 3 \sigma^{2} 4 \sigma^{2} 1 \pi^{4} 5 \sigma^{2}$. The Hartree-Fock wavefunction at the equilibrium internuclear separation of $2.132 \mathrm{au}$ was obtained by a SCF calculation using a standard [9s5p] primitive Cartesian Gaussian function contracted to a [4s3p] basis set (Dunning 1970). With this basis set, the total energy of the ground state is $-112.6869 \mathrm{au}$, close to the HF limit of $-112.7860 \mathrm{au}$ (Huo 1965).

In these studies we obtained the continuum wavefunctions for $l \leqslant 10$ and $m \leqslant 2$. In the calculations, the partial-wave expansion of the direct and exchange potentials are carried out up to $l=38$ and $l=19$ respectively. Only one iteration was needed in the Schwinger iterative procedure.

Calculations have been carried out in the $20-50 \mathrm{eV}$ range. To determine the energy of the scattered electron for a given incident energy, we used the experimental values (Krupenie 1966) of the vertical excitation energy from the $\nu=0$ vibrational level of the initial electronic state.

\subsection{Excitation to the triplet states}

The $\mathrm{a}^{\prime}{ }^{3} \Sigma^{+}$and $\mathrm{d}^{3} \Delta$ excited states are essentially characterised by the single-electron transition from the occupied $1 \pi$ orbital to the unoccupied $2 \pi$ orbital, whereas the $a^{3} \Pi$ state corresponds to the transition from the $5 \sigma$ to $2 \pi$ orbital. The experimental vertical excitation energies for the $\mathrm{a}^{\prime}{ }^{3} \Sigma^{+}, \mathrm{d}^{3} \Delta$ and a ${ }^{3} \Pi$ states are 8.2, 9.1 and $6.3 \mathrm{eV}$ (Krupenie 1966) respectively. For these states, the $2 \pi$ orbital is taken as an eigenfunction of the $V_{N-1}$ potential formed by removing an electron from the $1 \pi$ orbital for the $\mathrm{a}^{\prime 3} \Sigma^{+}$and $\mathrm{d}^{3} \Delta$ states and from the occupied $5 \sigma$ orbital for the a ${ }^{3} \Pi$ state. The calculations were carried out using the computer codes developed by Goddard and co-workers (Hunt and Goddard 1974). With the same Gaussian basis set as in the ground-state SCF calculation, we obtain vertical excitation energies of $7.65,8.55$ and $5.80 \mathrm{eV}$ for these states respectively. 
Electron impact excitation of the triplet states in $\mathrm{CO}$ involves only the exchange part of the distorted-wave matrix elements. These matrix elements are again calculated by use of the single-centre expansion of the distorted waves, the target orbitals and the Coulomb interaction. In this expansion, sufficient terms are included to ensure the convergence of all matrix elements. The resulting radial two-electron integrals are evaluated numerically by Simpson's rule. The single-centre expansion of the distorted-wave matrix elements converges rapidly for exchange-type excitation processes. In this calculation, we included partial-wave matrix elements for $l, l^{\prime} \leqslant 10$ and $m, m^{\prime} \leqslant 2$. No higher order corrections were included. We estimate that the contribution from those higher partial-wave matrix elements to the differential cross sections to be less than $5 \%$ at the impact energies of this work.

For excitations of the $\mathrm{a}^{\prime}{ }^{3} \Sigma^{+}$and a ${ }^{3} \Pi$ states, we used the Franck-Condon factors calculated by Nicholls (1962). However, for the transition $X^{1} \Sigma \rightarrow d^{3} \Delta$, we are not aware of any published Franck-Condon factors. Hence for this transition, we calculated the Franck-Condon factors using the published RKR potentials for both the ground and excited states (Krupenie and Weissman 1965), using the Numerov method (Cooley 1961) to integrate the radial equation.

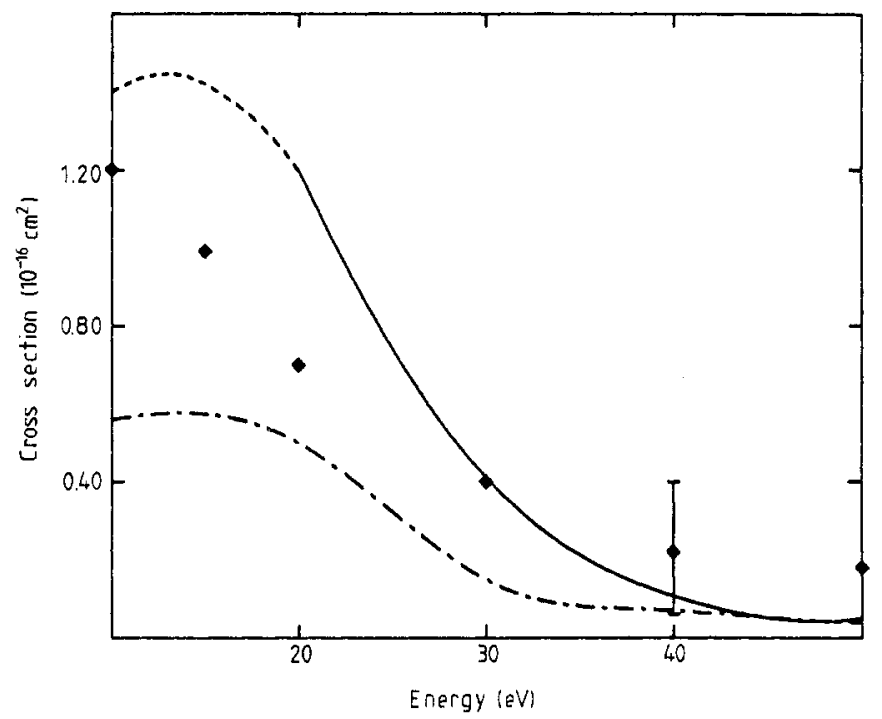

Figure 1. Integral cross section for the excitation of the a ${ }^{3} \Pi$ state: the calculated $D W$ results (full curve); the extrapolated DW results (broken curve, see text); the BOR results of Chung and Lin (1974) (chain curve) and experimental data of Ajello (1971) (diamonds).

In figure 1 , we compare the integral cross section for the excitation of the $a^{3} \Pi$ state obtained by the DW method with those measured by emission experiments (Ajello 1971) along with the theoretical results obtained by the Born-Ochkur-Rudge (BOR) approximation (Chung and Lin 1974). The agreement between the DW cross sections and emission results is reasonable at the incident energies about $20 \mathrm{eV}$. Below that energy the shape resonance in the elastic scattering wavefunction leads to a large unphysical value of the DW inelastic cross section similar to the $\mathrm{N}_{2}$ case (Lee and McKoy $1982 \mathrm{a}, \mathrm{b}$ ). The resonance-like feature in these DW inelastic cross sections is simply a consequence of the ${ }^{2} \Pi$ shape resonance, which occurs around $3.8 \mathrm{eV}$, in 
e-CO collisions at the static-exchange level. In the present DW model the final-state elastic wave is strongly affected by the resonance behaviour at $3.8 \mathrm{eV}$. This enhancement is a serious limitation of the present DW model. In figure 1 and other figures the broken lines below $20 \mathrm{eV}$ do not correspond to DW values but are shown just to indicate the probable shape of the cross section in that region. The BOR approximation shows a smaller cross section at lower energies. As expected, the DW and BOR cross sections agree well as the energy increases.

In figure 2, we show the DW differential cross sections at 20 and $50 \mathrm{eV}$, along with the experimental measurements of Cartwright and Trajmar (1981) at $20 \mathrm{eV}$. Although it reproduces the shape of the experimental DCs quite well, the DW method overestimates the magnitude of the DCs by a factor of four. This overestimation of the DCS at $20 \mathrm{eV}$ is probably caused by the influence of the elastic shape resonance at low energies and hence we can expect better agreement with experiment at higher incident energies.

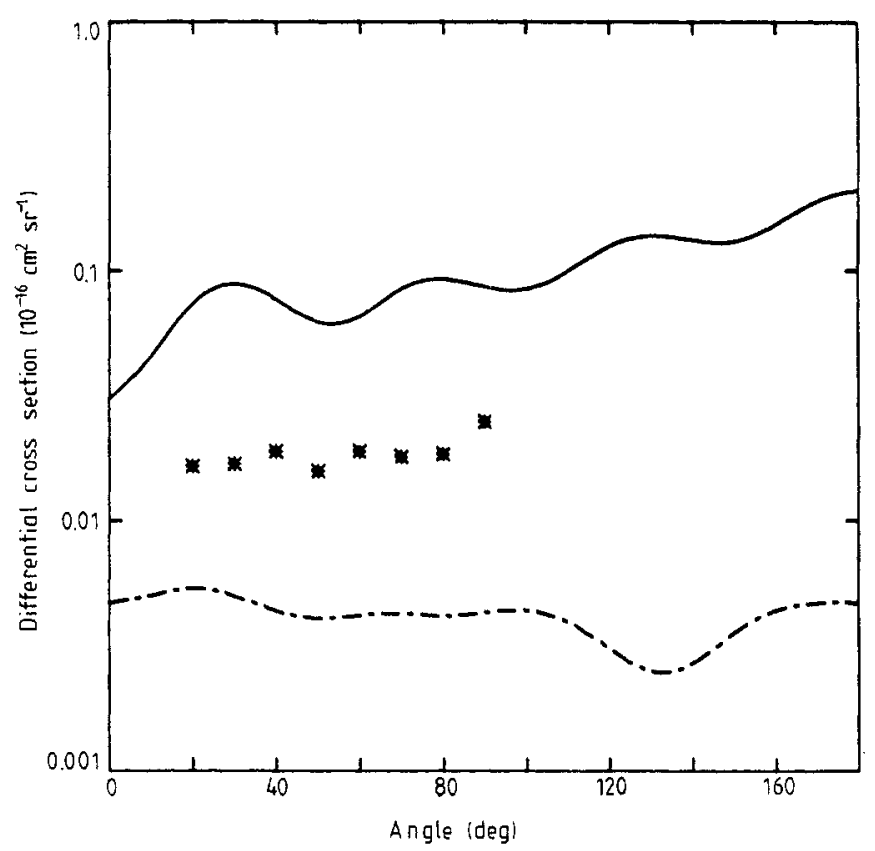

Figure 2. DCs for the excitation of the $a^{3} \Pi$ state: the DW results at $20 \mathrm{eV}$ (full curve); the experimental data of Cartwright and Trajmar (1981) at $20 \mathrm{eV}$ (stars); the Dw results at $50 \mathrm{eV}$ (chain curve).

In figure 3, we compare our DW integral cross sections for excitation of the $\mathrm{a}^{\prime}{ }^{3} \Sigma^{+}$ state with the BOR results (Chung and Lin 1974). In this case, the BOR method gives considerably larger cross sections than the DW method. Although there are no experimental measurements available for this state, the analysis of the measured electron transport coefficient data (Land 1978) suggests that the BOR data should be reduced by a factor of 0.35 . This reduced BOR cross section is also shown in figure 3. The agreement between this reduced BOR data and the DW cross section is quite good. In figure 3, we have also shown the DW (Lee and McKoy 1982a, b) and experimental (Cartwright et al 1977a,b) integral cross sections for excitation of the $A^{3} \Sigma_{u}^{+}$state of $N_{2}$. One sees that the energy dependence of the $N_{2}$ cross sections is 


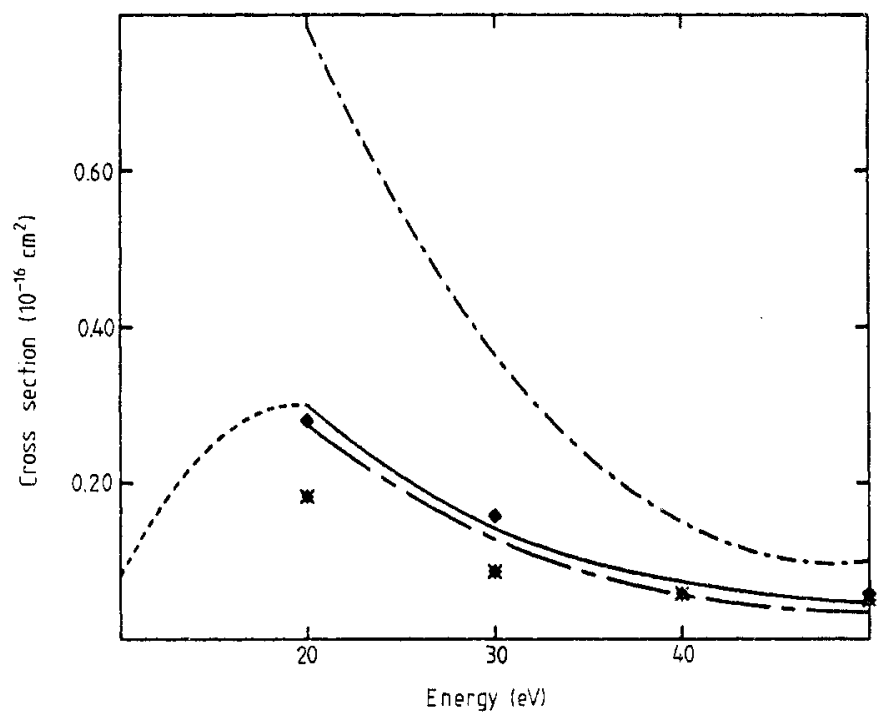

Figure 3. Integral cross section for the excitation of the $\mathrm{a}^{\prime}{ }^{3} \Sigma^{+}$state: the calculated DW results (full curve); the extrapolated DW results (broken curve, see text); the BOR results of Chung and Lin (1974) (chain curve); the BOR results multiplied by 0.35 as suggested by Land (1978) (long and short dashed curve); the DW results for excitation of the $A^{3} \Sigma_{u}^{+}$ state of $\mathrm{N}_{2}$ (Lee and McKoy 1982a) (diamonds) and the corresponding experimental data for $\mathrm{N}_{2}$ (Cartwright et al 1977b) (stars).

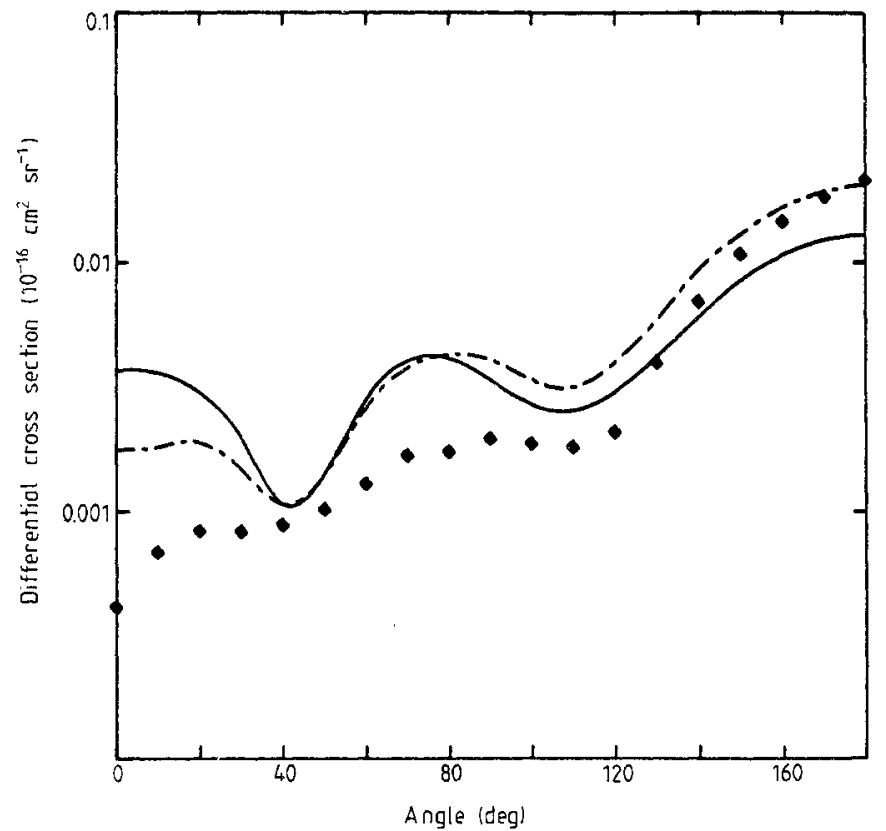

Figure 4. DCS for excitation of the $\mathrm{a}^{\prime}{ }^{3} \Sigma^{+}$state: the $\mathrm{DW}$ results at $50 \mathrm{eV}$ (full curve); the DW result for the corresponding excitation in $\mathrm{N}_{2}$ (chain curve, see text), the experimental DCS of the corresponding excitation in $\mathrm{N}_{2}$ (Cartwright et al 1977b) (diamonds). 
similar to that of $\mathrm{CO}$, while the magnitude of the cross section in $\mathrm{N}_{2}$ is smaller by a factor of two at $20 \mathrm{eV}$, but getting closer to the $\mathrm{CO}$ cross section at $50 \mathrm{eV}$.

In figure 4 , we show the differential cross section for excitation of the $\mathrm{a}^{\prime}{ }^{3} \Sigma^{+}$state of $\mathrm{CO}$ at $50 \mathrm{eV}$. Again there are no experimental DCs available for this state. We have also plotted the DW (Lee and McKoy 1982a, b) and experimental (Cartwright et al 1977a, b) DCS for excitation of the $\mathrm{A}^{3} \Sigma_{\mathrm{u}}^{+}$state in $\mathrm{N}_{2}$ at the same energy. The agreement between these two sets of theoretical cross sections is quite good both in shape and magnitude. The same comparison has been done for elastic scattering of electrons by $\mathrm{N}_{2}$ and CO (Bromberg 1969, 1970, Dubois and Rudd 1976, Tanaka et al 1978) showing that the angular distributions of elastically scattered electrons are very similar for these two gases. This similarity in behaviour is reasonable for the elastic scattering cross section since $\mathrm{N}_{2}$ and $\mathrm{CO}$ are isoelectronic. It is interesting to observe that similar behaviour also occurs in the inelastic cross sections.

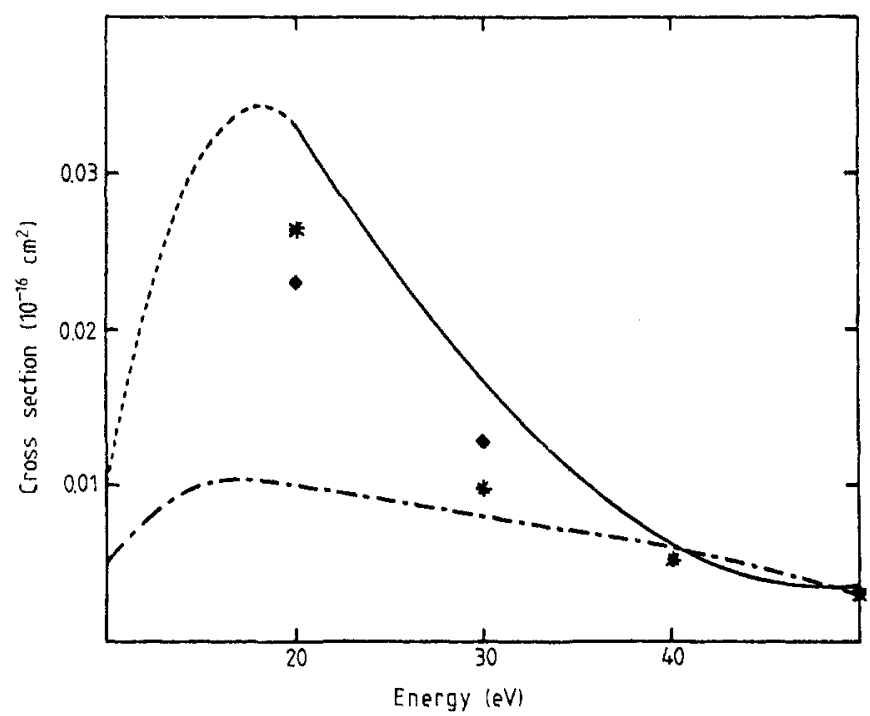

Figure 5. Integral cross sections for excitation of the $d^{3} \Delta$ state: the calculated DW results (full curve); the extrapolated DW results (broken curve); the BOR results of Chung and Lin (1974) multiplied by ten (chain curve); the DW results of the corresponding excitation in $\mathrm{N}_{2}$ (diamonds); the experimental data for the corresponding excitation in $\mathrm{N}_{2}$ (stars).

In figure 5 we show that Dw integral cross section for excitation of the $\mathrm{d}^{3} \Delta$ state along with the BOR cross sections of Chung and Lin (1974). No experimental data are available for this state. The agreement between these two sets of theoretical data is poor. Part of this poor agreement could be due to the fact that in their BOR calculation Chung and Lin (1974) used the virtual $2 \pi$ orbital of the ground-state Hartree-Fock calculation for the excited $2 \pi$ orbital of $d^{3} \Delta$ state. Again comparison shows that the DW integral cross sections for $\mathrm{CO}$ are quite similar to those for excitation of the $\mathrm{W}^{3} \Delta_{\mathrm{u}}$ state in $\mathrm{N}_{2}$ for incident energies above $15 \mathrm{eV}$, i.e., away from the elastic shape resonance region. The DCs for excitation of the $\mathrm{d}^{3} \Delta$ state at $50 \mathrm{eV}$ is shown in figure 6. In this case, there are also no experimental measurements available. Comparison with the DW (Lee and McKoy 1982a, b) nnd experimental (Cartwright et al $1977 \mathrm{a}, \mathrm{b}$ ) DCS for excitation of the $\mathrm{W}^{3} \Delta_{\mathrm{u}}$ state in $\mathrm{N}_{2}$ at the same energy shows these two DCs are similar, particularly in the forward and backward direction. 


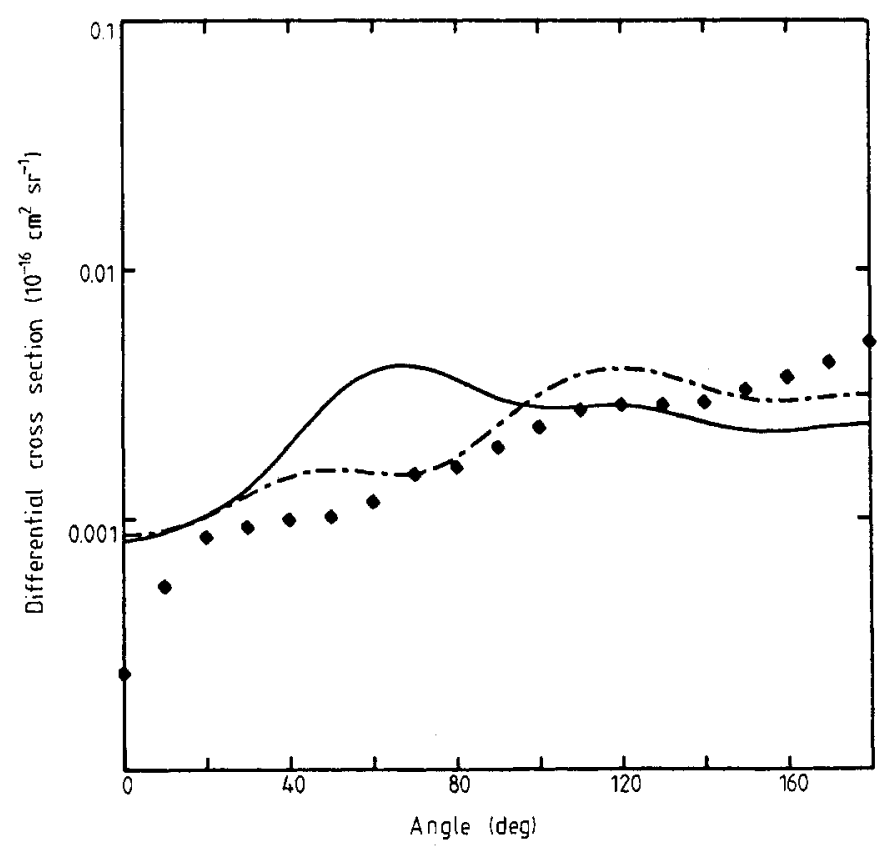

Figure 6. DCS for excitation of the $\mathrm{d}^{3} \Delta$ state: the labels are the same as in figure 4 .

\subsection{Excitation of the singlet states}

The $\mathrm{A}^{1} \Pi$ and $\mathrm{D}^{1} \Delta$ excited states essentially correspond to the single-electron transition from the occupied $5 \sigma$ and $1 \pi$ orbitals to the unoccupied $2 \pi$ orbital respectively. The experimental excitation energies for the $\mathrm{A}^{1} \Pi$ and $\mathrm{D}^{1} \Delta$ states are 8.35 and $10.4 \mathrm{eV}$ respectively. For these states, single-configuration wavefunctions are also obtained in the same way as for the triplet excited states and with the same basis set as in the SCF calculation. We obtained vertical excitation energies of 9.10 and $9.95 \mathrm{eV}$ for the $\mathrm{A}^{1} \Pi$ and $\mathrm{D}^{1} \Delta$ states respectively.

In these singlet-singlet excitation processes, both the direct and exchange parts of the partial-wave transition matrix elements are included with $l, l^{\prime} \leqslant 10$ and $m, m^{\prime} \leqslant 2$. The contribution from higher partial-wave terms to the direct matrix elements are included in the Born approximation in the same way as described by Fliflet and McKoy (1980). No higher-order corrections are carried out in the exchange matrix elements.

For excitation of the $\mathrm{A}^{1} \Pi$ state, we also used the Franck-Condon factors calculated by Nicholls (1962). For the excitation of the $\mathrm{D}^{1} \Delta$ state, we calculated the FranckCondon factors using the RKR potential curve for both the ground and excited states (Krupenie and Weissman 1965, Simmons and Tilford 1966).

In figure 7, we compare the DW integral cross sections for excitation of the $\mathrm{A}^{1} \Pi$ state with the BOR results (Chung and Lin 1974) and the experimental data obtained by the emission method (Ajello 1971, Mumma et al 1971). The agreement between these two sets of theoretical results is good for impact energies above $30 \mathrm{eV}$. However, both results overestimate the cross sections by a factor of three, compared with the experimental data from the emission measurements. Below $30 \mathrm{eV}$, the resonance-like feature is again observed and causes a large enhancement of the cross sections in the DW method. 


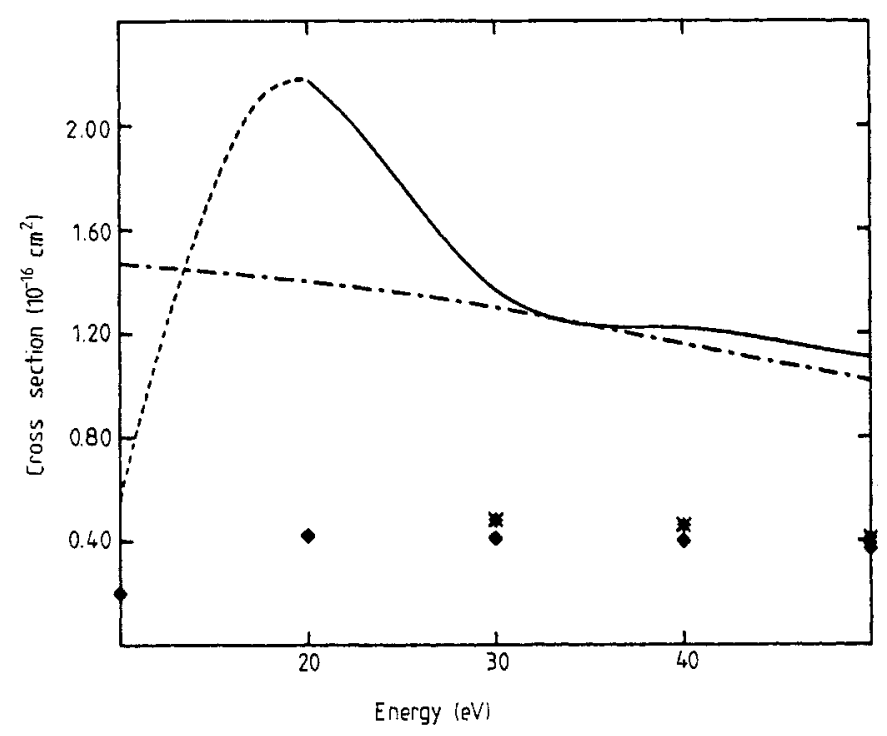

Figure 7. Integral cross sections for excitation of the $A^{1} \Pi$ state: the calculated $D w$ results (full curve); the extrapolated DW results (broken curve); the BOR results of Chung and Lin (1974) (chain curve); the experimental data of Ajello (1971) (diamonds) and Mumma et al (1971) (stars).

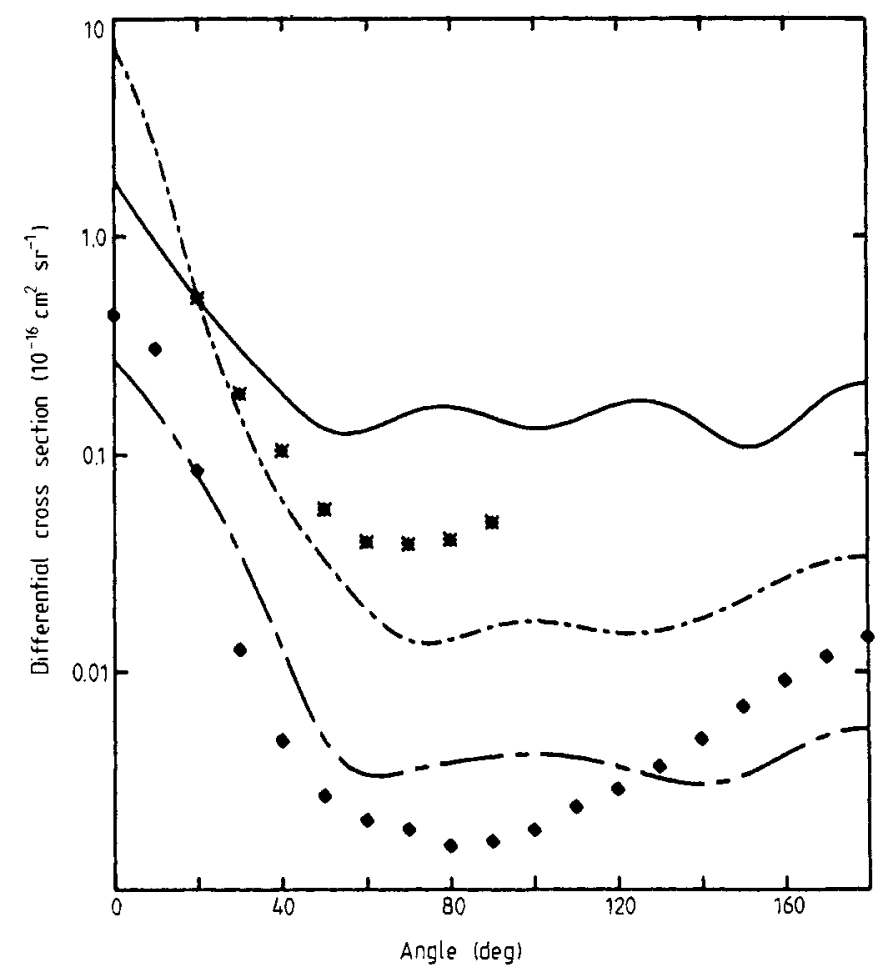

Figure 8. DCS for excitation of the $A^{1} \Pi$ state: the DW results at $20 \mathrm{eV}$ (full curve) and $50 \mathrm{eV}$ (chain curve); the experimental data at $20 \mathrm{eV}$ (Cartwright and Trajmar 1981) (stars); the DW (Lee and McKoy 1982b) (long and short dashed curve) and experimental DCs (Chutjian et al 1983) (diamonds) for the corresponding excitation in $\mathrm{N}_{2}$ at $50 \mathrm{eV}$. 
In figure 8, we show the DW DCs for this excitation at 20 and $50 \mathrm{eV}$. At $20 \mathrm{eV}$, we compare our DCS with the experimental results of Cartwright and Trajmar (1981) in the angular range between 20 and $90^{\circ}$. The Dw theory again reproduces the shape of the experimental DCS. However, the theory overestimates its magnitude. As in the excitation of the $\mathrm{A}^{3} \Pi$ state, this overestimation is caused by the enhancement of the cross section due to the shape resonance in the ${ }^{2} I I$ channel in the elastic scattering.

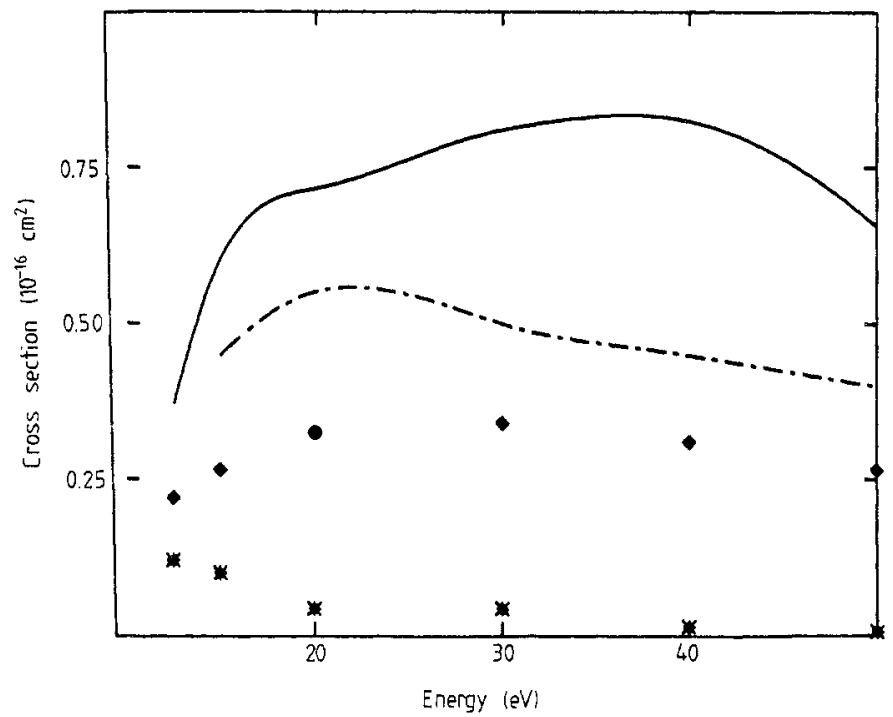

Figure 9. Integral cross sections for excitation of the $\mathrm{D}^{1} \Delta$ states: the labels are the same as in figure 5.

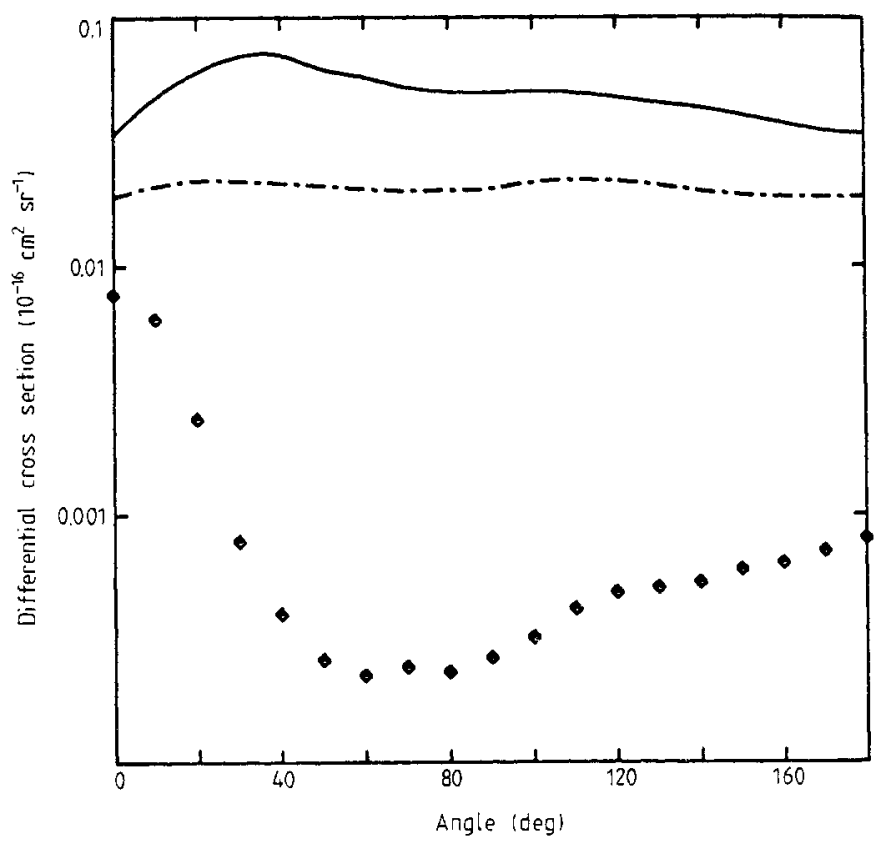

Figure 10. DCs for excitation of the $\mathrm{D}^{1} \Delta$ state: the labels are the same as in figure 6 . 
We expect that better agreement might be obtained at higher impact energies. At $50 \mathrm{eV}$, we compare the DCs for this excitation with the DW (Lee and McKoy 1982a, b) and experimental (Cartwright et al 1977a, b) DCs of the corresponding excitation (i.e., $\mathrm{X}^{1} \Sigma_{\mathrm{g}}^{+} \rightarrow \mathrm{a}{ }^{1} \Pi_{\mathrm{g}}$ ) in $\mathrm{N}_{2}$. In this case, the shape of these two sets of theoretical DCS is very similar, although the magnitude of the DCS in $\mathrm{CO}$ is much larger.

In figure 9 , we show the $\mathrm{DW}$ integral cross sections for excitation of the $\mathrm{D}^{1} \Delta$ state along with the BOR results of Chung and Lin (1974). The agreement between these results is again very poor. The comparison with the DW integral cross sections for excitation of the $\mathrm{w}^{1} \Delta_{\mathrm{u}}$ state in $\mathrm{N}_{2}$ shows the same angular dependence but the cross section for $\mathrm{CO}$ is larger in general by a factor of two. This difference between the present DW results and those of the BOR can be partly due again to the use of the $2 \pi$ virtual orbital of the ground state in the $w^{1} \Delta_{u}$ state in the BOR calculation. However, comparison with the data of $\mathrm{N}_{2}$ suggests that interchannel coupling may be important in the excitation of this state.

The DCs for excitation of the $\mathrm{D}^{1} \Delta$ state at $50 \mathrm{eV}$ is compared with the DW (Lee and McKoy 1982a, b) and experimental (Cartwright et al 1977a, b) DCs of the corresponding excitation of $\mathrm{N}_{2}$ in figure 10 . The poor agreement between the calculated and measured cross sections for this state of $\mathrm{N}_{2}$ has been discussed previously (Lee and McKoy 1982a).

\section{Conclusion}

We have applied the distorted-wave approximation to calculate the electron impact cross sections for several states of $\mathrm{CO}$. Comparison with measured cross sections for the ${ }^{3} \Pi$ and $A^{1} \Pi$ states shows that, although the Dw method overestimates the magnitude of the DCS by a factor of four, it does much better in reproducing the shape of the DCS. There are no experimental data on the DCS available for any other states of CO. The DW integral cross section for the $A^{1} \Pi$ is also about three times larger than the measured cross sections. In general, however, the DW cross sections differ substantially from those predicted by the BOR approximation. As expected the difference between these DW and BOR cross sections decreases with increasing impact energies. Comparison of these cross sections with those of the corresponding excitations in $\mathrm{N}_{2}$ show, that in most cases, the shapes of the angular distribution are similar for these two systems. In general, the DCs of $\mathrm{CO}$ are larger. As in the case of $\mathrm{N}_{2}$, the large enhancement of the DW inelastic cross sections caused by the shape resonance in the ${ }^{2} \Pi$ elastic out-going distorted wave is observed. These resonance-like enhancements limit the applicability of the DW theory at energies near threshold. Simple procedures for removing these resonance-like enhancements and to extend the DW method to more complicated molecules (e.g., $\mathrm{CO}_{2}$ ) are now in progress.

\section{Acknowledgments}

We thank Professor W A Goddard III for making some of his computer codes available to us. These results are based upon work supported by the National Science Foundation (Office of International Programs (US-Brasil)) under grant No INT 80-01473. One of us (LMT) acknowledges the financial support of FAPESP (Saõ Paulo, Brasil). The authors also thank Dr R R Lucchese for many fruitful discussions and Dr S Trajmar for providing experimental data prior to publication. 
The research reported in this paper made use of the CRAY-1 computer of the National Center of Atmospheric Research (NCAR, Boulder, Colorado) which is supported by the National Science Foundation. Part of the calculations were done on the Dreyfus-NSF Theoretical Chemistry computer which was funded through grants from the Camille and Henry Dreyfus Foundation, the National Science Foundation (grant No CHE-7820235) and the Sloan Fund of the California Institute of Technology.

\section{References}

Aarts J F M and de Heer F J $1970 J$. Chem. Phys. 52 5354-9

Ajello J M $1971 \mathrm{~J}$. Chem. Phys. 55 3158-68

Arrighini G P, Biondi F, Guidotti C, Giagi A and Marinelli F 1980 Chem. Phys. 52 133-41

Bromberg J P 1969 J. Chem. Phys. 50 3906-21

$1970 \mathrm{~J}$. Chem. Phys. 52 1243-7

Cartwright D C 1970 Phys. Rev. A 2 1331-48 1972 Phys. Rev. A 51974

Cartwright D C, Chutjian A, Trajmar S and Williams W 1977a Phys. Rev. A 16 1013-40

1977 b Phys. Rev. A 16 1041-51

Cartwright D C and Trajmar S 1981 private communication

Chung S and Lin C C 1972 Phys. Rev. A 6 988-1002

- 1974 Phys. Rev. A 9 1954-64 1978 Phys. Rev. A 17 1874-91

Chung S, Lin C C and Lee E T P 1975 Phys. Rev. A 12 1340-9

Chutjian A, Cartwright D C and Trajmar S 1977 Phys. Rev. A 16 1052-60

- 1983 Phys. Rep, and private communication

Cooley J W 1961 Math. Comput. 15 363-74

Corrigan S J B 1965 J. Chem. Phys. 43 4381-6

Dubois R D and Rudd M E 1976 J. Phys. B: At. Mol. Phys. 9 2657-67

Dunning T H 1970 J. Chem. Phys. 53 2823-33

Finn T G and Doering J P 1976 J. Chem. Phys. 64 4490-4

Fliflet A W and McKoy V 1980 Phys. Rev. A 21 1863-75

Fliflet A W, McKoy V and Rescigno T N 1980 Phys. Rev. A 21 788-92

Hazi A V 1981 Phys. Rev. A 23 2232-40

Holley T K, Chung S, Lin C C and Lee E T P 1981 Phys. Rev. A 24 2946-52

Hunt W J and Goddard W A III 1974 Chem. Phys. Lett. 24 464-71

Huo W M 1965 J. Chem. Phys. 43 624-46

Khare S P 1966 Phys. Rev. $14933-7$

1967 Phys. Rev. 157 107-12

Krupenie P H 1966 The Band Spectrum of Carbon Monoxide NSRDS-NBS 5 (Washington, DC: US Govt Printing Office)

Krupenie P H and Weissman S 1965 J. Chem. Phys. 43 1529-34

Land J E 1978 J. Appl. Phys. 49 5716-21

Lee M T, Lucchese R R and McKoy V 1982 Phys. Rev. A to be published

Lee M T and McKoy V 1982a Phys. Rev. A submitted for publication

1982 b manuscript in preparation

Linder F and Schmidt H 1971 Z. Naturf. 26a 1617-25

Lucchese R R and McKoy V 1979 J. Phys. B: At. Mol. Phys. 12 1421-4

Mumma M J, Stone E J and Zipf E C 1971 J. Chem. Phys. 54 2627-34

Nicholls R W 1962 J. Quant. Spectrosc. Radiat. Transfer 2 433-49

Rice J K 1968 PhD Thesis California Institute of Technology

Rescigno T N, McCurdy C W and McKoy V 1975 J. Phys. B. At. Mol. Phys. 8 1433-6

Rescigno T N, McCurdy C W, McKoy V and Bender C F 1976 Phys. Rev. A 13 216-23

Sawada T, Sellin D L and Green A E S 1972 J. Geophys. Res. 77 4819-28

Simmons J D and Tilford S G 1966 J. Chem. Phys. 45 2965-8

Srivastava S K and Jensen S 1977 J. Phys. B: At. Mol. Phys. 10 3341-6 
Tanaka H, Srivastava S K and Chutjian A 1978 J. Chem. Phys. 69 5329-33

Trajmar S, Cartwright D C, Rice J K, Brinkman R T and Kupperman A 1968 J. Chem. Phys. 49 5464-71

Trajmar S, Cartwright D C and Williams W 1971 Phys. Rev. A 4 1482-92

Watson D K, Lucchese R R, McKoy V and Rescigno T N 1980 Phys. Rev. A 21 738-44

Yung Y L, Gladstone G R, Chang K M, Ajello J M and Srivastava S K 1982 Ap. J. 254 L65-9 